\title{
THE RATE OF INCREASE OF MEAN VALUES OF FUNCTIONS IN WEIGHTED HARDY-ORLICZ SPACES
}

BY

\section{AJAY K. SHARMA and AMBIKA BHAT}

\begin{abstract}
Let $0 \leq q<\infty$. For each $f$ in the weighted Hardy-Orlicz space $H_{\Phi, q}$ we show that $\frac{d\left\|f_{r}\right\|_{\Phi, q}}{d r}$ grows at most like $o(1 / 1-r)$ as $r \rightarrow 1$.

Mathematics Subject Classification 2010: 30H10, 46E10, 30D55.

Key words: weighted Hardy space, weighted Hardy-Orlicz space.
\end{abstract}

\section{Introduction and preliminaries}

Let $\mathbb{D}$ be the open unit disk in the complex plane $\mathbb{C}, \partial \mathbb{D}$ the unit circle, $H(\mathbb{D})$ the class of all holomorphic functions on $\mathbb{D}$ and $d m(\theta)=\frac{1}{2 \pi} d \theta$ be the normalized Lebesgue measure on $\partial \mathbb{D}$. For $0<p<\infty$ and $0 \leq q<\infty$, define

$$
\left\|f_{r}\right\|_{p, q}=\left(\frac{1}{2 \pi} \int_{0}^{2 \pi}\left|f\left(r e^{i \theta}\right)\right|^{p}\left(1-r^{2}\right)^{q} d \theta\right)^{1 / p}
$$

The weighted Hardy space $H_{p, q}(\mathbb{D})=H_{p, q}$ is the family of all holomorphic functions $f$ in $\mathbb{D}$ satisfying $\|f\|_{p, q}=\sup _{0<r<1}\left\|f_{r}\right\|_{p, q}<\infty$. It is trivial that $H_{p, 0}$ is just the classical Hardy space $H^{p}$ and $H_{p, q}$ is a Banach space for $p \geq 1$. We write $\|f\|_{p}$ for $\|f\|_{p, 0}$. For more about $H^{p}$ and $H_{p, q}$ see [3], [10] and [11]. Let $S T^{2}(\mathbb{R})$ denote the class of strongly convex functions $\Phi$ : $[-\infty, \infty) \rightarrow[0, \infty)$ (that is, $\Phi$ is non-negative, convex and non-decreasing with $\frac{\Phi(t)}{t} \rightarrow \infty$ as $\left.t \rightarrow \infty\right)$ which satisfy:

(i) $\Phi(t)=0$ for all $t<0$ with $\Phi(0)=\Phi^{\prime}(0)=0$;

(ii) $\Phi^{\prime \prime}(t)$ exists for all $t>0$; 
(iii) $\Phi(2 t) \leq C \Phi(t)$ for some positive constant $C$ and for all $t>0$.

For example, the function $\Phi:[-\infty, \infty) \rightarrow[0, \infty)$ defined as

$$
\Phi(x)= \begin{cases}x^{2}, & \text { if } x \geq 0 \\ 0, & \text { if } x<0\end{cases}
$$

is in $S T^{2}(\mathbb{R})$. For $\Phi \in S T^{2}(\mathbb{R})$, we define the Hardy-Orlicz space $H^{\Phi}$ by

$$
H^{\Phi}=\left\{f \in H(\mathbb{D}): \sup _{0<r<1} \int_{\partial \mathbb{D}} \Phi\left(\log ^{+}\left|f\left(r e^{i \theta}\right)\right|\right) d m<\infty\right\}
$$

where

$$
\log ^{+}(x)= \begin{cases}\log x, & \text { if } x \geq 1 \\ 0, & \text { if } x<1\end{cases}
$$

Although the integral expression above does not define a norm in $H^{\Phi}$, the distance $d(f, g)=\int_{\partial \mathbb{D}} \Phi\left(\log ^{+}\left|f\left(r e^{i \theta}\right)-g\left(r e^{i \theta}\right)\right|\right) d m$ defines a translationinvariant metric on $H^{\Phi}$, and turns $H^{\Phi}$ into a complete metric space. Obviously, the inequalities $\log ^{+} x \leq \log (1+x) \leq 1+\log ^{+} x, x \geq 0$ and $2 \log ^{+} x \leq \log \left(1+x^{2}\right) \leq 1+2 \log ^{+} x, x \geq 0$ and the fact $\Phi$ is nondecreasing convex function imply that $\Phi\left(\log ^{+} x\right) \leq \Phi(\log (1+x)) \leq \Phi(1+$ $\left.\log ^{+} x\right) \leq \frac{1}{2} \Phi(2)+\frac{1}{2} \Phi\left(2 \log ^{+} x\right) \leq \frac{1}{2} \Phi(2)+\frac{1}{2} C \Phi\left(\log ^{+} x\right)$ and $\Phi\left(\log ^{+} x\right) \leq$ $\Phi\left(2 \log ^{+} x\right) \leq \Phi\left(\log \left(1+x^{2}\right)\right) \leq \Phi\left(1+2 \log ^{+} x\right) \leq \frac{1}{2} \Phi(2)+\frac{1}{2} \Phi\left(4 \log ^{+} x\right) \leq$ $\frac{1}{2} \Phi(2)+\frac{1}{2} C \Phi\left(\log ^{+} x\right)$. Hence $f \in H^{\Phi}$ if and only if $\sup _{0<r<1} \int_{\partial \mathbb{D}} \Phi(\log (1+$ $\left.\left.\left|f\left(r e^{i \theta}\right)\right|\right)\right) d m<\infty$ or if and only if $\sup _{0<r<1} \int_{\partial \mathbb{D}} \Phi\left(\log \left(1+\left|f\left(r e^{i \theta}\right)\right|^{2}\right) d m<\right.$ $\infty$. For more about Hardy-Orlicz spaces see [6], [7] and [9]. For $0 \leq q<\infty$ and $\Phi \in S T^{2}(\mathbb{R})$, defined weighted Hardy-Orlicz space $H_{\Phi, q}$ as

$$
H_{\Phi, q}=\left\{f \in H(\mathbb{D}): \sup _{0 \leq r<1} \int_{\partial \mathbb{D}} \Phi\left(\log \left(1+\left|f\left(r e^{i \theta}\right)\right|^{2}\right)\right)\left(1-|r|^{2}\right)^{q} d m<\infty\right\} .
$$

Abusing notation, we will write

$$
\|f\|_{\Phi, q}=\sup _{0<r<1} \int_{\partial \mathbb{D}} \Phi\left(\log \left(1+\left|f\left(r e^{i \theta}\right)\right|^{2}\right)\right)\left(1-|r|^{2}\right)^{q} d m<\infty .
$$

Note that $H_{\Phi, 0}$ reduces to the Hardy-Orlicz space $H_{\Phi}$.

It is well known that $\left\|f_{r}\right\|_{p}$ is a non-decreasing function of $r$. Furthermore, HARDY [4] showed that $\log \left\|f_{r}\right\|_{p}$ is a convex function of $\log r$. Other 
properties of mean values of analytic functions can be found in [1], [2] and [8]. Recently, MARHREGHI [5] showed that $\frac{d\left\|f_{r}\right\|_{p}}{d r}$ grows at most like $o(1 / 1-r)$ as $r \rightarrow 1$. This result was extended to weighted Hardy spaces by XIONG and LIU [10]. In this paper, we generalized this result to weighted Hardy-Orlicz spaces.

Throughout this paper constants are denoted by $C$, they are positive and not necessarily the same at each occurrence. The $O(1)$ and $o(1)$ are usual Landau symbols.

\section{Some lemmas}

In this section, we prove some lemmas that are helpful in the proof of main result of this paper. Suppose that $f \neq 0$ is in $H(\mathbb{D})$. Let $W(z)=$ $\Phi\left(\log \left(1+|f(z)|^{2}\right)\right)\left(1-|z|^{2}\right)^{q}$ for $0 \leq q<\infty$, and $\nabla$ be the gradient operator.

Lemma 2.1. Let $0<r \leq 1$ and $D_{r}=\{z:|z|<r\}$. For $z_{0} \in D_{r}$ and small $\varepsilon>0$, let $D\left(z_{0}, \varepsilon\right)=\left\{z:\left|z-z_{0}\right| \leq \varepsilon\right\} \subset D_{r}$ and

$$
I_{\varepsilon}=\int_{\partial D\left(z_{0}, \varepsilon\right)}\left(\log (r /|z|) \frac{\partial W}{\partial n}-W \frac{\partial \log (r /|z|)}{\partial n}\right) d l .
$$

If $z_{0} \neq 0$ is a zero of $f$, then $\lim _{\varepsilon \rightarrow 0} I_{\varepsilon}=0$. If $z_{0}=0$, then $\lim _{\varepsilon \rightarrow 0} I_{\varepsilon}=$ $2 \pi \Phi\left(\log \left(1+|f(0)|^{2}\right)\right)$.

Proof. Since $W(z)=\Phi\left(\log \left(1+|f(z)|^{2}\right)\right)\left(1-|z|^{2}\right)^{q}$, direct computation gives

$\nabla W(z)=\left(1-|z|^{2}\right)^{q} \nabla \Phi\left(\log \left(1+|f(z)|^{2}\right)\right)+\Phi\left(\log \left(1+|f(z)|^{2}\right)\right) \nabla\left(1-|z|^{2}\right)^{q}$

and

$$
\left|\nabla \Phi\left(\log \left(1+|f(z)|^{2}\right)\right)\right| \leq 2\left|\Phi^{\prime}\left(\log \left(1+|f(z)|^{2}\right)\right)\right|\left|f(z) \| f^{\prime}(z)\right|\left|\frac{1}{\left(1-|f(z)|^{2}\right)}\right| .
$$

So

$$
\begin{aligned}
& \left|\frac{\partial W(z)}{\partial n}\right| \leq|\nabla W(z)| \leq\left(1-|z|^{2}\right)^{q}\left|\nabla \Phi\left(\log \left(1+|f(z)|^{2}\right)\right)\right| \\
& +\Phi\left(\log \left(1+|f(z)|^{2}\right)\right)\left|\nabla\left(1-|z|^{2}\right)^{q}\right| \\
& \leq 2\left(1-|z|^{2}\right)^{q}\left|\Phi^{\prime}\left(\log \left(1+|f(z)|^{2}\right)\right)\right||f(z)|\left|f^{\prime}(z)\right|\left|\frac{1}{\left(1+|f(z)|^{2}\right)}\right| \\
& +2\left|\Phi\left(\log \left(1+|f(z)|^{2}\right)\right)\right| q|z|\left(1-|z|^{2}\right)^{q-1}
\end{aligned}
$$


and $\left|\frac{\partial \log (r /|z|)}{\partial n}\right| \leq|\nabla \log (r /|z|)|=\frac{1}{|z|}$. Write

$$
\begin{aligned}
I_{\epsilon} & =\int_{\partial D\left(z_{0}, \varepsilon\right)}\left(\log (r /|z|) \frac{\partial W}{\partial n}-W \frac{\partial \log (r /|z|)}{\partial n}\right) d l \\
& =\int_{0}^{2 \pi} \log \left(r /\left|z_{0}+\varepsilon e^{i \theta}\right|\right) \frac{\partial W\left(z_{0}+\varepsilon e^{i \theta}\right)}{\partial n} \varepsilon d \theta-\int_{\partial D\left(z_{0}, \varepsilon\right)} W(z) \frac{\partial \log (r /|z|)}{\partial n} d l \\
& =I_{1}-I_{2} .
\end{aligned}
$$

For convenience, let $C>0$ be a constant independent of $\varepsilon$. If $z_{0} \neq 0$ is a zero of order $k \geq 1$, then

$$
I_{1}=\int_{0}^{2 \pi} \log \left(r /\left|z_{0}+\varepsilon e^{i \theta}\right|\right) \frac{\partial W\left(z_{0}+\varepsilon e^{i \theta}\right)}{\partial n} d \theta \leq O(1) \varepsilon
$$

and $I_{2} \leq O(1) \varepsilon$. Taking limit $\varepsilon \rightarrow 0$ we get $\lim _{\varepsilon \rightarrow 0} I_{2}=0$. Hence $\lim _{\varepsilon \rightarrow 0} I_{\varepsilon}=0$.

If $z_{0}=0$ and $f(z) \neq 0$, then $I_{1}=\int_{0}^{2 \pi} \log \left(r /\left|\varepsilon e^{i \theta}\right|\right) \frac{\partial W\left(\varepsilon e^{i \theta}\right)}{\partial n} \varepsilon d \theta \leq O(1) \varepsilon$. Taking limit $\varepsilon \rightarrow 0$ we get $\lim _{\varepsilon \rightarrow 0} I_{1}=0$.

Similarly, $I_{2}=\int_{0}^{2 \pi} W\left(\varepsilon e^{i \theta}\right) d \theta=\left(1-\varepsilon^{2}\right)^{q} \int_{0}^{2 \pi} \Phi\left(\log \left(1+\left|f\left(\varepsilon e^{i \theta}\right)\right|^{2}\right)\right) d \theta$. Taking limit $\varepsilon \rightarrow 0$ we get $\lim _{\varepsilon \rightarrow 0} I_{2}=2 \pi \Phi\left(\log \left(1+|f(0)|^{2}\right)\right.$ ). Hence $\lim _{\varepsilon \rightarrow 0} I_{\varepsilon}$ $=2 \pi \Phi\left(\log \left(1+|f(0)|^{2}\right)\right)$.

Lemma 2.2. Let $f \in H_{\Phi, q}(\mathbb{D}), 0 \leq q<\infty$ and $f \neq 0$. Then

$$
\begin{aligned}
\lim _{r \rightarrow 1} \int_{0}^{2 \pi} \mid & \left(\log \left(1+\left|f\left(r e^{i \theta}\right)\right|^{2}\right)\right) \mid\left(1-|z|^{2}\right)^{q} d \theta \\
& =2 \pi \Phi\left(\log \left(1+|f(0)|^{2}\right)\right)+\int_{|z|<1} \log (1 /|z|) G(z) d x d y
\end{aligned}
$$

where $G(z)=\nabla^{2}\left|\Phi\left(\log \left(1+|f(z)|^{2}\right)\right)\right|\left(1-|z|^{2}\right)^{q}+2 \nabla\left|\Phi\left(\log \left(1+|f(z)|^{2}\right)\right)\right| \nabla(1-$ $\left.|z|^{2}\right)^{q}+\left|\Phi\left(\log \left(1+|f(z)|^{2}\right)\right)\right| \nabla^{2}\left(1-|z|^{2}\right)^{q}$.

Proof. Since $f \neq 0$ is analytic in $\mathbb{D}$, for any $0<R<1$, we can choose $r, R<r<1$ such that $f$ has finite many zeros in $D_{r}=\{|z|<r\}$ and no zeros on the circle $\partial D_{r}$. Let $\left\{z_{1}, z_{2}, \cdots z_{n}\right\}$ be the set consisting of 0 and all zeros of $f$ in $D_{r}$. Take $\varepsilon>0$ so small that $\left\{z:\left|z-z_{k}\right|<\varepsilon\right\} \subset D_{r}$ for $1 \leq k \leq n$ and all these disks are disjoint.

Denote $\Omega=D_{r} \backslash \bigcup_{k=1}^{n}\left\{z:\left|z-z_{k}\right|<\epsilon\right\}$. The function $W(z)=\Phi(\log (1+$ $\left.\left.|f(z)|^{2}\right)\right)\left(1-|z|^{2}\right)^{q}$ is twice differentiable in a neighborhood of $\bar{\Omega}$. Then by Green's theorem we have

$$
\int_{\Omega} \log (r /|z|) \nabla^{2} W(z) d x d y=\int_{\partial \Omega}\left(\log (r /|z|) \frac{\partial W}{\partial n}-W \frac{\partial \log (r /|z|)}{\partial n}\right) d l
$$


Direct computation gives $\nabla^{2} W(z)=\nabla^{2}\left|\Phi\left(\log \left(1+|f(z)|^{2}\right)\right)\right|\left(1-|z|^{2}\right)^{q}+$ $2 \nabla\left|\Phi\left(\log \left(1+|f(z)|^{2}\right)\right)\right| \nabla\left(1-|z|^{2}\right)^{q}+\left|\Phi\left(\log \left(1+|f(z)|^{2}\right)\right)\right| \nabla^{2}\left(1-|z|^{2}\right)^{q}=G(z)$. The direction of every small circle in $\partial \Omega$ is clockwise. For $\varepsilon \rightarrow 0$, by Lemma 2.1 formula $(2.1)$ becomes $\int_{\partial D_{r}}\left(\log (r /|z|) \frac{\partial W}{\partial n}-W \frac{\partial \log (r /|z|)}{\partial n}\right) d l=$ $2 \pi \Phi\left(\log \left(1+|f(0)|^{2}\right)\right)+\int_{D_{r}} \log (r /|z|) G(z) d x d y$. The left-side of above formula is equal to $\int_{0}^{2 \pi} W\left(\varepsilon e^{i \theta}\right) d \theta-2 \pi \Phi\left(\log \left(1+|f(0)|^{2}\right)\right)$. Proceeding to limit as $r \rightarrow 1$, through a sequence $\left(r_{n}\right)$ such that $f$ has no zeros on $\partial D_{r_{n}}$, accomplish the proof.

\section{The rate of increase of $\left\|f_{r}\right\|_{\Phi, q}$}

Theorem 3.1. Let $\Phi \in S T^{2}(\mathbb{R})$ and $0 \leq q<\infty$. Let $f \in H_{\Phi, q}(\mathbb{D})$ and $f \neq 0$. Then $\frac{d\left\|f_{r}\right\|_{\Phi, q}}{d r}=o(1 / 1-r)$ as $r \rightarrow 1$.

Proof. As in the proof of Lemma 2.2, we choose suitable $r$ and $\varepsilon$. Replacing $\log (r /|z|)$ by $\log (1 /|z|)$ and using Green's theorem, we have

$$
\int_{\Omega} \log (r /|z|) \nabla^{2} W(z) d x d y=\int_{\partial \Omega}\left(\log (1 /|z|) \frac{\partial W}{\partial n}-W \frac{\partial \log (r /|z|)}{\partial n}\right) d l .
$$

For $\varepsilon \rightarrow 0$, using Lemma 2.1 for $r=1$, formula (3.1) turns into

$$
\begin{aligned}
& 2 \pi \Phi\left(\log \left(1+|f(0)|^{2}\right)\right)+\int_{D_{r}} \log (1 /|z|) \nabla^{2} G(z) d x d y \\
& =\int_{\partial D_{r}}\left(\log (1 /|z|) \frac{\partial W}{\partial n}-W \frac{\partial \log (1 /|z|)}{\partial n}\right) d l \\
& =\int_{0}^{2 \pi}\left(\log (1 / r) \frac{\partial W\left(r e^{i \theta}\right)}{\partial r}-\frac{W\left(r e^{i \theta}\right)}{r}\right) r d \theta \\
& =\int_{0}^{2 \pi} W\left(r e^{i \theta}\right) d \theta-r \log r \int_{0}^{2 \pi} \frac{\partial W\left(r e^{i \theta}\right)}{\partial r} d \theta \\
& =\int_{0}^{2 \pi}\left|\Phi\left(\log \left(1+\left|f\left(r e^{i \theta}\right)\right|^{2}\right)\right)\right|\left(1-r^{2}\right)^{q} d \theta-2 \pi r \log r \frac{d\left\|f_{r}\right\|_{\Phi, q}}{d r} .
\end{aligned}
$$

Now let $r \rightarrow 1$, through a sequence $\left(r_{n}\right)$ such that $f$ has no zeros on $\partial D_{r_{n}}$, by Lemma 2.2, we have $\lim _{r \rightarrow 1} \log r \frac{d\left\|f_{r}\right\|_{\Phi, q}}{d r}=0$. Hence, $\lim _{r \rightarrow 1} \frac{d\left\|f_{r}\right\|_{\Phi, q}}{d r}=$ $o(1 / \log r)=o(1 / 1-r)$. 
Corollary 3.2. Let $\Phi \in S T^{2}(\mathbb{R})$ and $0 \leq q<\infty$. Let $f \in H_{\Phi}(\mathbb{D})$ and $f \neq 0$. Then $\frac{d\left\|f_{r}\right\|_{\Phi}}{d r}=o(1 / 1-r)$ as $r \rightarrow 1$.

Corollary 3.3. If $f \in H_{\Phi, q}(\mathbb{D})$ with $0 \leq q<\infty$, then we have

$$
\begin{aligned}
2 \lim _{r \rightarrow 1} \int_{0}^{2 \pi} \Phi & \left(\log \left(1+\left|f\left(r e^{i \theta}\right)\right|^{2}\right)\right)\left(1-r^{2}\right)^{q} d \theta \\
= & \int_{\mathbb{D}}\left(1-|z|^{2}\right)^{q} \nabla^{2}\left(\left|\Phi\left(\log \left(1+|f(z)|^{2}\right)\right)\right|\left(1-|z|^{2}\right)^{q}\right) d x d y \\
& +4 \int_{\mathbb{D}}\left|\Phi\left(\log \left(1+|f(z)|^{2}\right)\right)\right|\left(1-|z|^{2}\right)^{q} d x d y
\end{aligned}
$$

Proof. Denote

$$
J_{\varepsilon}=\int_{\partial D\left(z_{0}, \varepsilon\right)}\left(\left(1-|z|^{2}\right) \frac{\partial W}{\partial n}-W \frac{\partial\left(1-|z|^{2}\right)}{\partial n}\right) d l,
$$

where $z_{0}$ is a zero of $f$. Similar arguments as in the proof of Lemma 2.1, we have $\lim _{\varepsilon \rightarrow 0} I_{\varepsilon}=0$. Choosing suitable $r$ and $\varepsilon$ as in the proof of Theorem 3.1, by Green's theorem, we have

$$
\begin{array}{r}
\int_{\Omega}\left(1-|z|^{2}\right)^{q} \nabla^{2} W(z)-\nabla^{2}\left(1-|z|^{2}\right) W(z) d x d y \\
=\int_{\partial D \Omega}\left(\left(1-|z|^{2}\right) \frac{\partial W}{\partial n}-W \frac{\partial\left(1-|z|^{2}\right)}{\partial n}\right) d l .
\end{array}
$$

As $\varepsilon \rightarrow 0$ above formula turns into

$$
\begin{aligned}
\int_{D_{r}} & \left(1-|z|^{2}\right)^{q} \nabla^{2} W(z)+4 W(z) d x d y \\
& =\int_{\partial D_{r}}\left(\left(1-|z|^{2}\right) \frac{\partial W}{\partial n}-W \frac{\partial\left(1-|z|^{2}\right)}{\partial n}\right) d l \\
& =\int_{0}^{2 \pi}\left(\left(1-r^{2}\right) \frac{\partial W\left(r e^{i \theta}\right)}{\partial n}+2 r W\left(r e^{i \theta}\right)\right) r d \theta .
\end{aligned}
$$

By Theorem 3.1, we have

$$
\lim _{r \rightarrow 1} \int_{0}^{2 \pi} r\left(1-r^{2}\right) \frac{\partial W\left(r e^{i \theta}\right)}{\partial n} d \theta=2 \lim _{r \rightarrow 1}\left[\left(1-r^{2}\right) \frac{\partial}{d r} \int_{0}^{2 \pi} W\left(r e^{i \theta}\right) d \theta\right]=0 .
$$

The proof is completed by letting $r \rightarrow 1$, through a sequence $\left(r_{n}\right)$ such that $f$ has no zeros on $\partial D_{r_{n}}$ in formula (3.3). 
Note that

$$
\begin{aligned}
\nabla^{2}\left(\left|\Phi\left(\log \left(1+|f(z)|^{2}\right)\right)\right|=\right. & 4\left[\Phi^{\prime \prime}\left(\log \left(1+|f(z)|^{2}\right)\right)|f(z)|^{2}\right. \\
& \left.+\Phi^{\prime}\left(\log \left(1+|f(z)|^{2}\right)\right)\right] \frac{\left|f^{\prime}(z)\right|^{2}}{1+|f(z)|^{2}}
\end{aligned}
$$

Taking $q=0$ in formula (3.2) we obtain

$$
\begin{aligned}
\int_{0}^{2 \pi} & \left(\left|\Phi\left(\log \left(1+\left|f\left(r e^{i \theta}\right)\right|^{2}\right)\right)\right| d \theta\right. \\
& =2 \int_{\mathbb{D}}\left(1-|z|^{2}\right)\left[\Phi^{\prime \prime}\left(\log \left(1+|f(z)|^{2}\right)\right)|f(z)|^{2}+\Phi^{\prime}\left(\log \left(1+|f(z)|^{2}\right)\right)\right] \\
& \frac{\left|f^{\prime}(z)\right|^{2}}{1+|f(z)|^{2}} d x d y+2 \int_{\mathbb{D}}\left|\Phi\left(\log \left(1+|f(z)|^{2}\right)\right)\right| d x d y
\end{aligned}
$$

which is reminiscent of Hardy-Stein identity for Hardy-Orlicz space.

Acknowledgments. We are extremely thankful to the referee for his/her comments and suggestions. This work is a part of the research project sponsored by National Board of Higher Mathematics (NBHM)/DAE, India (Grant No. 48/4/2009/R\& D-II/426).

\section{REFERENCES}

1. Beckenbach, E.F. - A property of mean values of an analytic function, Rend. Circ. Mat. Palermo, 1 (1952), 157-163.

2. Beckenbach, E.F.; Gustin, W.; Shniad, H. - On the mean modulus of an analytic function, Bull. Amer. Math. Soc., 55 (1949), 184-190.

3. Duren, P.L. - Theory of $H^{p}$ Spaces, Pure and Applied Mathematics, 38, Academic Press, New York-London, 1970.

4. HARdy, G.H. - The mean value of the modulus of an analytic function, Proc. London Math. Soc., 14 (1915), 269-277.

5. Mashreghi, J. - The rate of increase of mean values of functions in Hardy spaces, J. Aust. Math. Soc., 86 (2009), 199-204.

6. Sharma, A.K.; Sharma, S.D. - Compact composition operators on Hardy-Orlicz spaces, Mat. Vesnik, 60 (2008), 215-224. 
7. Sharma, A.K.; UeKI, S. - Compactness of composition operators acting on weighted Bergman-Orlicz spaces, Ann. Polon. Math., 103 (2011), 1-13.

8. ShNiAd, H. - Convexity properties of integral means of analytic functions, Pacific J. Math., 3 (1953), 657-666.

9. Stoll, M. - A characterization of Hardy-Orlicz spaces on planar domains, Proc. Amer. Math. Soc., 117 (1993), 1031-1038.

10. Xiong, C.; LiU, J. - The rate of increase of mean values of functions in weighted Hardy spaces, Acta Math. Vietnam., 37 (2012), 89-93.

11. Zhu, K.H. - Operator Theory in Function Spaces, Monographs and Textbooks in Pure and Applied Mathematics, 139, Marcel Dekker, Inc., New York, 1990.

Received: 27.III.2012

Revised: 2.X.2012

Accepted: 3.X.2012
School of Mathematics, Shri Mata Vaishno Devi University, Kakryal, Katra-182320, J\& K, INDIA

aksju_76@yahoo.com ambikabhat.20@gmail.com 